\title{
Prophylaxe und Therapie von PONV
}

Univ.-Prof. Dr. Peter Kranke widmete seinen Vortrag am Kongress der Deutschen Gesellschaft für Anästhesiologie und Intensivmedizin (DGAI) der jüngst veröffentlichten US-Leitlinie zu postoperativer Übelkeit und Erbrechen (PONV).

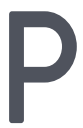

ONV ist gemeinsam mit postoperativen Schmerzen eine maßgebliche Determinante von Anästhesiequalität aus Sicht der Patient*innen. 'Die vierte KonsensusGuideline zu deren Management trägt diesem Umstand Rechnung und ersetzt damit die Vorläuferversion aus dem Jahr 2014. „Es gibt ganz klar einen Trend hin zu multimodaler Prophylaxe", bestätigt OA Univ.-Prof. Dr. Peter Kranke, MBA, vom Universitätsklinikum Würzburg, der sich selbst seit Jahrzehnten mit PONV beschäftigt und CoAutor der Leitlinie ist.

\section{EPIDEMIOLOGIE}

Die Relevanz der Implikation einer generellen Prophylaxe beruht laut Prof. Kranke nicht nur auf medizinischen, sondern ebenso auf juristischen Konsequenzen. Aus diversen Publikationen bekannte und zum Teil diskrepante

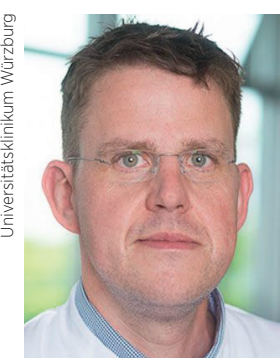

OA Univ.-Prof. Dr.

Peter Kranke, MBA

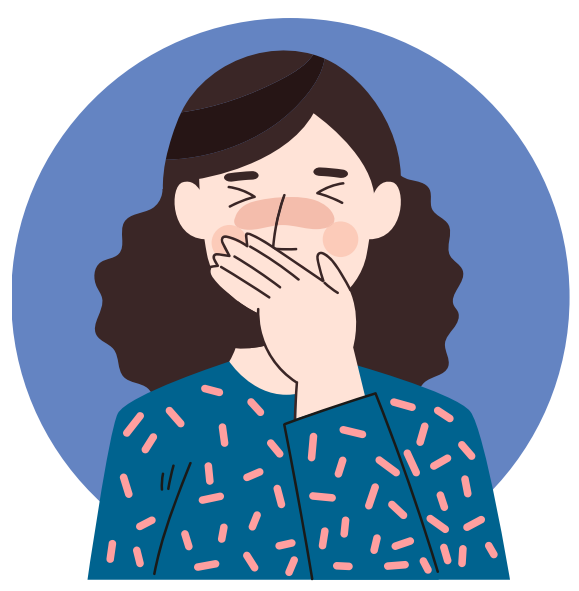

auch auf Seite der Anästhesist*innen, wie beispielsweise Dauer der OP, Alter, Geschlecht, Art des Eingriffs, postoperative Opioide, Raucherstatus und positive Anamnese für PONV. Dabei zeigte sich, dass die früher praktizierte Gewichtung nicht wesentlich besser als ein simpler Score-Wert zwischen 0-4 ist (vgl. Tabelle). Bei ein bis zwei Risikofaktoren wird eine multimodale Risikoprophylaxe mit zwei verschiedenen Substanzen empfohlen, sind es mehr als zwei, sollen drei bis vier Antiemetika verabreicht werden. Zudem ist in der Leitlinie ein eigenes Schema für Kinder abgebildet, wobei sich das Risikoprofil in niedrig, moderat und hoch unterteilt. In Summe sind demnach die Applikation zweier Antiemetika zur Prophylaxe, eingebettet in ein Konzept mit weiteren Interventionen und Adjustierungen falls notwendig, ein guter Startpunkt. Nicht vergessen werden sollen überdies

\section{Tabelle: PONV-Score Würzburg}

\begin{tabular}{|lc|}
\hline Risikofaktoren & Punkte \\
\hline weibliches Geschlecht & 1 \\
\hline Nichtraucher & 1 \\
\hline $\begin{array}{l}\text { Anamnese für PONV } \\
\pm \text { Reisekrankheit }\end{array}$ & 1 \\
\hline postoperative Opioide & 1 \\
\hline Summe & $0-4$ \\
\hline
\end{tabular}

*PONV-Risiko bei 0 Punkte: $10 \%$,

1 Punkt: $20 \%$, 2 Punkte: $40 \%$, 3 Punkte: $60 \%$, 4 Punkte: $80 \%$
Möglichkeiten zur Risikominimierung, wie die Vermeidung von hochdosiertem Neostigmin und volatilen Anästhetika im Allgemeinen, aber auch die Abklärung einer eventuellen Regionalanästhesie und der sparsame Gebrauch von postoperativen Opioiden mithilfe einer multimodalen Analgesie.

\section{SUBSTANZUNTERSCHIEDE}

Zusätzlich zu Listen mit für Erwachsene und die pädiatrische Population geeigneten Antiemetika einschließlich körpergewichtsadaptierten Dosisvorschlägen macht die neue Guideline Angaben zur Evidenz sowie zum optimalen Zeitpunkt der Applikation. Bezüglich der Wirksamkeit der einzelnen Substanzen hob Kranke eine rezente Cochrane-Metaanalyse hervor, die, mit 585 eingeschlossenen Studien und 97.516 Patient*innen, absolute Zahlen zu deren Effektivität nennt und diese einem Ranking unterzieht. ${ }^{4}$

PONV ist ein bedeutsamer Faktor für die zeitnahe postoperative Erholung. Je mehr Risikofaktoren vorliegen, desto mehr antiemetische Substanzen sollten prophylaktisch verabreicht werden. Erfreulicherweise lässt sich international ein Trend von restriktiver hin zu einer liberalen PONV-Prophylaxe feststellen, der gemäß der vorliegenden KonsensusGuideline unterstützt wird. „Betreiben Sie ausreichend Prophylaxe und denken Sie auch im Aufwachraum daran, die Patient*innen möglichst symptomfrei auf die Station zu entlassen, weil es dort in der Regel deutlich länger dauert, bis sie Symptomlinderung erfahren", schließt Kranke die Präsentation.

Bericht: Mag. Christopher Waxenegger

Referenzen:

1 Hocking G et al. Br J Anaesth. 2013; 111(6):979989

2 Gan TJ et al. Anesth Analg. 2020; 131(2):411-448 3 Apfel et al. Anesthesiology 2012; 117:475-486

4 Weibel S et al. Cochrane Database Syst Rev. 2020; 10(10):CD012859

Quelle: 23. Hauptstadtkongress der Deutschen Gesellschaft für Anästhesiologie und Intensivmedizin (DGAI); Symposium: Leitlinien auf dem Bierdeckel, 16.-17. September 2021, Berlin, VIRTUELL 